Document downloaded from:

http://hdl.handle.net/10251/142519

This paper must be cited as:

Lajara Vizcaino, JR.; Perez Solano, JJ.; Pelegrí Sebastiá, J. (11-2). Predicting the Batteries' State of Health in Wireless Sensor Networks Applications. IEEE Transactions on Industrial Electronics. 65(11):8936-8945. https://doi.org/10.1109/TIE.2018.2808925

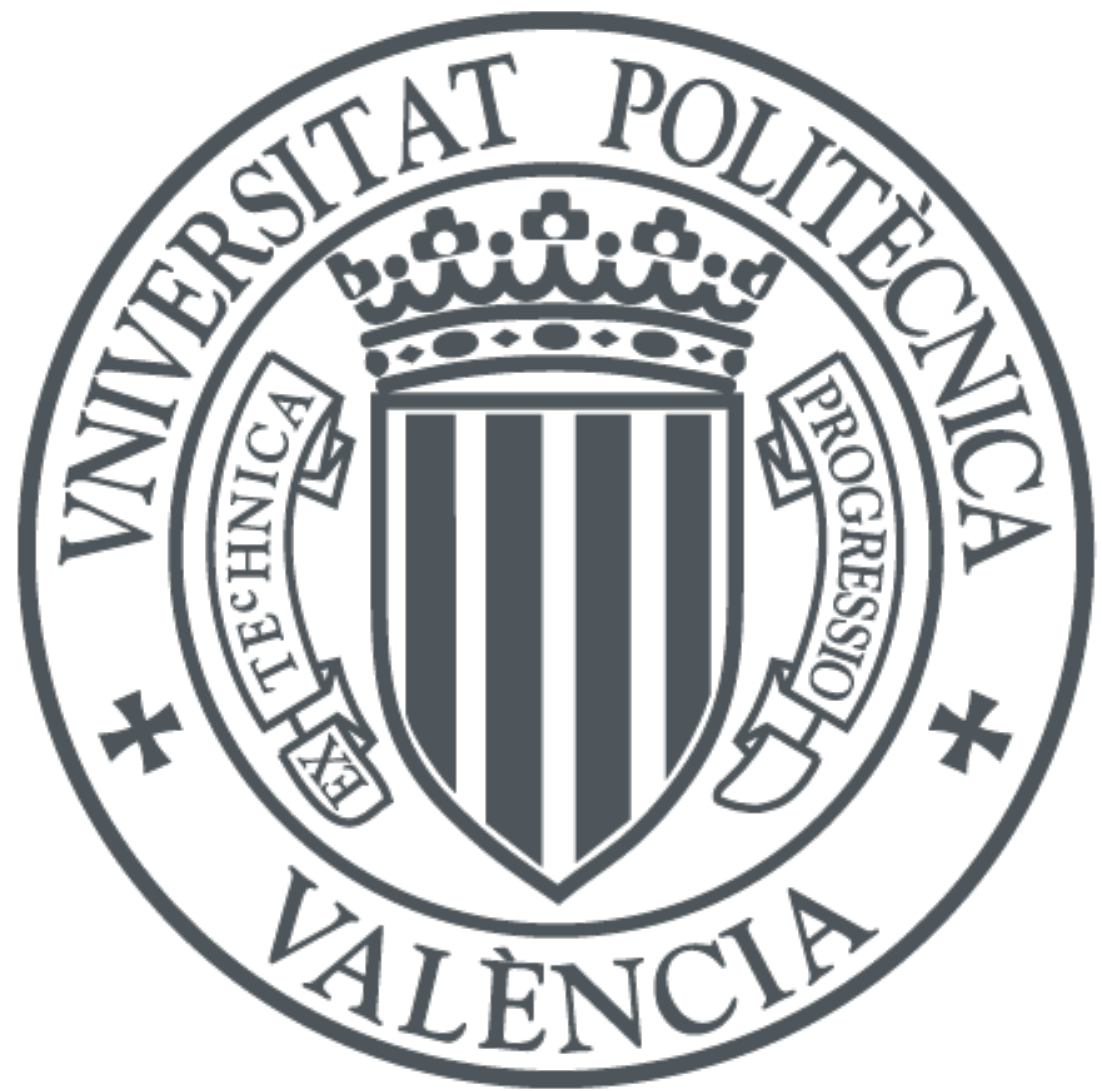

The final publication is available at

https://doi.org/10.1109/TIE.2018.2808925

Copyright Institute of Electrical and Electronics Engineers

Additional Information 


\section{Predicting the batteries State of Health in Wireless Sensor Networks applications}

\begin{abstract}
The lifetime of wireless sensor networks deployments depends strongly on the nodes battery state of health. It is important to detect promptly those motes whose batteries are affected and degraded by ageing, environmental conditions, failures, etc. There are several parameters that can provide significant information of the battery state of health, such as: the number of charge/discharge cycles, the internal resistance, voltage, drained current, temperature, etc. The combination of these parameters can be used to generate analytical models capable of predicting the battery state of health. The generation of these models needs a previous process to collect dense data traces with sampled values of the battery parameters during a large number of discharge cycles under different operating conditions. The collected data allow the development of mathematical models that can predict the battery state of health. These models are required to be simple because they must be executed in motes with low computational capabilities. The article shows the complete process of acquiring the training data, the models generation and its experimental validation using rechargeable batteries connected to Telosb motes. The obtained results provide significant insight of the battery state of health at different temperatures and charge/discharge cycles.
\end{abstract}

Index Terms- Wireless sensor networks, battery, energy, models, SoH, microcontrollers.

\section{INTRODUCTION}

$\mathrm{W}$ IRELESS Sensor Networks' (WSN) motes are equipped with low-cost components and they are usually supplied by batteries. Due to the strict constraints that the motes present in terms of computational capabilities and power consumption, WSN applications usually involve simple tasks. Typical actions that a mote performs are data sampling from integrated sensors and wireless data transmission. But the application of this technology in real deployments is usually limited by the mote's power consumption. The mote's battery normally represents its only source of energy and the discharge cycle restricts the mote's lifetime. In some deployments, it is possible to use alternative sources of energy to recharge the battery with the help of additional energyharvesting elements based on solar panels, wind turbines, piezoelectric generators, RF harvesters, etc [1]. However, rechargeable batteries are affected by a wide variety of factors that can have a strong impact on its performance, such as: environmental temperature, depth of discharge, number of charge/discharge cycles, ageing process, etc. In this sense, the batteries' state of charge (SoC) and state of health $(\mathrm{SoH})$ represent common parameters used to determine the current battery state, however the meaning and the information that they provide is different. Battery SoC is a measure of the amount of electrical energy stored and indicates the charge that is left. In WSNs, a network mote can use the SoC to estimate when the battery will be depleted. SoC can be expressed as a percentage of some reference, i.e., the capacity when the battery was completely charged. It can be estimated applying mathematical models generated using a combination of different battery parameters [2-4].

On the other hand, SoH can be considered as a metric to evaluate the battery performance and detect capacity and power losses that appear with the pass of time [5]. It is employed to evaluate the current state of an aged battery and to compare it with the state of a fresh battery. Thus, SoH can be defined as the relationship between the nominal capacity at present time and the nominal initial capacity of the battery.

SoH $=\frac{C_{\text {nominal capacity at present time }}}{C_{\text {nominal capacity at initial time }}} * 100 \%$

This parameter provides significant information to predict a battery malfunction and it can avoid network failures due to unexpected mote deaths. With the aim of detecting batteries that are degraded, it should be desirable that every mote could periodically monitor the current battery SoH. This information would allow the implementation of energy aware applications, in which the mote's operation can change depending on the battery state [6]. In addition, the central node of the network can collect this information and facilitate the maintenance of network, indicating when a battery replacement is required.

In this context, the selection of appropriate models to estimate and predict the current $\mathrm{SoH}$ is of primary importance. Batteries can be diagnosed measuring variations in some intrinsic parameters, such as the capacity or the cell internal impedance. In references [7][8], it is shown how the internal resistance of the battery can provide a significant insight of its state. Thus, an abrupt increase of the internal resistance evidences a deterioration of the battery $\mathrm{SoH}$ and the appearance of ageing effects. In addition to the internal resistance, there are other battery parameters, such as: state of charge (SoC), the operating temperature, the number of charge/discharge cycles, initial voltage, depth of discharge (DoD), current drained, etc, which can provide significant information about the battery SoH. Thus, the main objective of this paper and its principal novelty is the development of simple and accurate $\mathrm{SoH}$ models to detect the battery degradation in WSN motes. The generation of these models is based on the combination of different parameters that can be directly measured during the mote operation. An additional requirement is that these models must be simple, because they are executed in WSN motes with very low computational capabilities. 
The development of the models needs an initial acquisition of dense data traces of several battery parameters during a complete discharge cycle. The data acquisition has to be repeated at different temperatures and within a large interval of charge/discharge cycles to guarantee a thorough coverage of a wide range of battery operating conditions. It should be also noticed that the validity of the training data traces is restricted to one specific battery model and may require the gathering of a new data set if it is changed. The adoption of this method can be enormously facilitated with the development of an autonomous system that handles the initial data acquisition process. This test-bench allows an easy acquisition of long discharge data traces from batteries connected to a WSN mote during its normal operation. In this paper, a detailed presentation of the test-bench has been included and it can be replicated and applied to different batteries.

\section{RELATED WORK}

Nowadays, Li-ion batteries have become the preferable type of batteries for supplying a vast variety of handheld devices, such as mobile phones, laptops, cameras, etc. The main advantages of this battery technology are: high energy density, great efficiency and long life in terms of number of charge/discharge cycles [5]. However, the performance of Liion batteries decreases with the pass of time due to a combination of different ageing factors, such as: temperature, charge/discharge cycles, current and cut-off voltage, etc. The foundations of the battery ageing can be thermal, chemical or mechanical and the complete understanding of all the mechanisms involved in these processes is not an easy matter [9]. Battery management systems can estimate the SoH to predict battery failures and determine when its replacement is necessary. However, SoH is a parameter that is very difficult to estimate in real systems. Li-ion batteries follow internal complicated ageing mechanisms that degrade their capacity and modify the internal structure. To this end, there are in the literature a great variety of methods proposed to determine the battery SoH [5], most of them applied to the case of batteries for electric vehicles.

The first type of methods is based on electrochemical impedance spectroscopy, which involves experimental measures of the battery parameters [10][11]. They provide accurate models to estimate the ageing effects correlating the Li-ion battery impedance spectra with the SoH. However, their main drawback is the complexity of the measurement process, which makes these methods unsuitable for WSNs.

Other approaches try to describe battery ageing using equivalent circuit models [12] and monitoring directly the evolution of certain parameters, such as: the internal resistance [7][8] or the total available capacity measured using coulomb counting [13]. Adaptive methods include parameter identification techniques to correlate the battery electrochemical properties with the SoH. The adaptive system compares the predicted with the experimentally measured values to estimate the cell dynamics. In some cases, the parameter identification can be performed using least squares algorithms [14-16]. In this group, we can highlight the work presented in reference [14] that is focused on the development of SoH models using linear regression to estimate the equivalent constant time of the battery. This parameter is highly correlated with the normalized capacity that provides direct information of the battery SoH. Some other algorithms proposed in the literature to develop battery management systems for electric vehicles are based on Kalman filters [1719], genetic algorithms [20] or particle filters [21][22]. The main problem of these adaptive methods is that the computational cost may be excessive for WSN motes. There are other attempts to achieve battery models using electrochemical formulation expressed as partial differential equations [23]. In [24] authors propose a combined SoC/SoH estimation algorithm via an electromechanical model that only uses samples of the battery voltage and current. However, the grade of complexity of the mathematical expressions in these methods made their implementation prohibitive in low cost motes.

In the literature there are also methods that address $\mathrm{SoH}$ estimation considering it as a black-box problem. In this case, there is no attempt to achieve a full understanding of the battery ageing mechanism. Proposed models rely on machine learning techniques to predict the battery SoH under different operating conditions. Some machine learning algorithms employed are support vector machines [25] [26], neural networks [27][28], probabilistic neural networks [29], recurrent neural networks [30] and fuzzy identification [31]. Nevertheless, the accuracy of these methods depends on the availability of large data traces of the battery parameters to train the estimation models.

As a result, it can be concluded that there is a large number of references in the literature considering the important problem of battery management for electric vehicles [5]. However, to the best of our knowledge, there is a great lack of references in the literature that deal with the design of simple models for SoH estimation in WSN's motes. Thus, most of the aforementioned methods are too complex for being executed in low-cost microcontrollers. In addition, none of them has been generated or validated using parameters sampled during the discharge cycle of a battery connected to a mote running a typical WSN application. On the other hand, there are very few references that include a revision of the whole process, that is, how to perform the data acquisition, the battery modeling, the implementation in real motes and the final validation using battery data traces. Thus, in this work we present the methodology that has been followed to generate and validate the SoH models. The process implies a first step to collect traces of experimental data in a real test-bench, a second stage to generate the models and finally the assessments performed to validate them.

\section{SYSTEM DESIGN}

The aim of this section is to provide a detailed description of the specific elements included in the test-bench. The proposed system monitors and controls the battery charge/discharge process and samples some parameters during the mote operation. The system applies a prefixed number of charge/discharge cycles during the normal functioning of a mote connected to the monitored battery. The mote runs a 
typical WSN application performing common tasks in this sort of networks. Thus, the system reproduces the real behavior of a mote executing a complete WSN program and monitors the battery parameters during a complete discharge cycle. Once the mote runs out of battery, the system controller recharges it, without having to remove the battery from the test-bench, and saves the data traces in independent files.

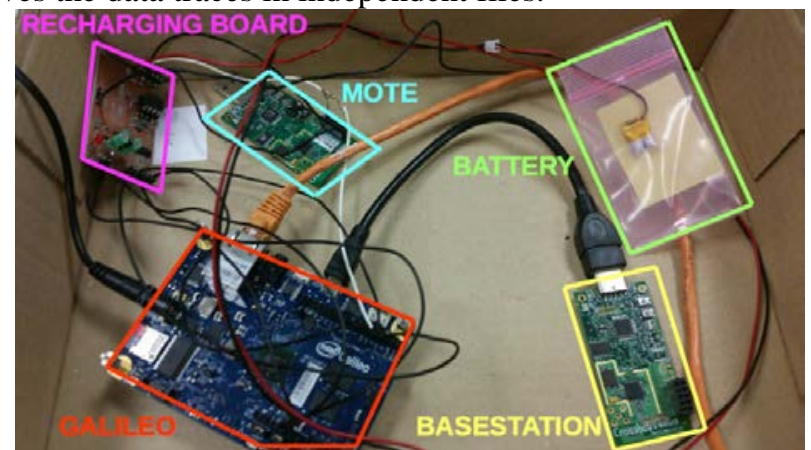

Fig. 1. Proposed system composed of four main blocks: Telosb mote, recharging board, Galileo board, Telosb base station and battery.

Most of the system components are built using open hardware elements, since it is intended to be widely accessible and easily reproducible. The test-bench is composed of three principal blocks: a) Telosb mote connected to a Li-ion battery, b) recharging board composed of a Li-ion battery charger and additional components for signal conditioning and c) Intel's Galileo board, which controls the system operation_and saves the data in a flash memory, with a Telosb basestation connected. The battery model selected is a rechargeable $3.7 \mathrm{~V}$ Li-ion Polymer Battery from Xtra-power with a capacity of 40 mAh [32]. An image of the system is shown in Fig. 1. Next, a detailed description of each block is provided.

\section{A. Mote}

In this work, the selected mote has been the well-known Telosb [33]. This mote features different power down modes. Thus, in active mode the mote drains 2,3 $\mathrm{mA}$, with the microcontroller running at $4 \mathrm{MHz}$, and $2.6 \mu \mathrm{A}$ when it is in standby [34]. The wireless transceiver increases the consumption to $19.7 \mathrm{~mA}$, when it is in reception, $17.4 \mathrm{~mA}$ in transmission, $426 \mu \mathrm{A}$ in sleep and $20 \mu \mathrm{A}$ in power down. The LEDs activation rises the current consumption in: a) blue LED $3.3 \mathrm{~mA}, \mathrm{~b})$ red LED $3.8 \mathrm{~mA}$ and c) green LED $5.3 \mathrm{~mA}$ [34]. Finally, the Sensirion SHT11 temperature sensor [35] consumes $2 \mu \mathrm{W}$ in sleep mode and $3 \mathrm{~mW}$ during its operation.

The battery parameters are sampled by the mote hardware, being the sampling process handled by the running program. The battery parameters measured are the internal supply voltage of the mote and the voltage at the battery terminals. The acquisition of the battery voltage is performed using the recharging board and sampling the Telosb_adc_input in Fig. 2. Taking into account these two sampled voltages and noticing that there is a shunt resistance of $10 \Omega$, the mote current consumption can be determined.

The program executed during the data collection process constitutes a typical WSN application. This program periodically wakes up the mote and samples the battery voltage, the microcontroller internal voltage and the temperature sensor. After that, it turns on the LEDs and the wireless transceiver to increase the power consumption. In this new power state, the mote acquires again the battery voltage and the internal supply voltage. Finally, it sends wirelessly the data to the basestation and goes to sleep. The acquisition of the battery voltage and the current drained at two different power states allows the estimation of the battery internal resistance using the equation [2]:

$$
R=\frac{V_{\text {low }}-V_{\text {high }}}{I_{\text {low }}-I_{\text {high }}}=\frac{\Delta V}{\Delta I}
$$

The specific list of states and actions performed with the corresponding employed time for this program is:

1. After the wake-up event the microcontroller resumes the program execution and measures the battery voltage, $\mathrm{V}$, and the internal Vcc (V_low, Vcc_low). The power mode of the mote in this state is active because only the microcontroller is active. This state lasts $275 \mathrm{~ms}$ and then the mote passes to state 2 .

2. The microcontroller measures the ambient temperature (Temp) and immediately passes to state 3 .

3. Switches-on the LEDs and the wireless transceiver to increase the power consumption. The power mode changes to high and mote goes to state 4 .

4. Mote measures the battery voltage, $\mathrm{V}$, and microcontroller Vcc (V_high, Vcc_high) in this high power mode. The mote stays at this state during 6 seconds and changes to state 5 afterwards.

5. Mote sends the sampled data using the wireless transceiver to the basestation mote and passes to state 6 .

6. Mote programs a sleep timer with a period of $700 \mathrm{~ms}$ and shuts-down everything: LEDs, transceiver and microcontroller. After this period, mote returns to state 1 .

This application has been programmed using TinyOS, which allows a rapid implementation of WSN applications.

\section{B. Recharging board}

The recharging board is an intermediate element situated between the Telosb mote and the Galileo platform that recharges the battery. The board integrates a Li-ion battery charger from Microchip, with reference MCP73831 [36]. Fig 2 depicts the board schematic and the expansion connectors.

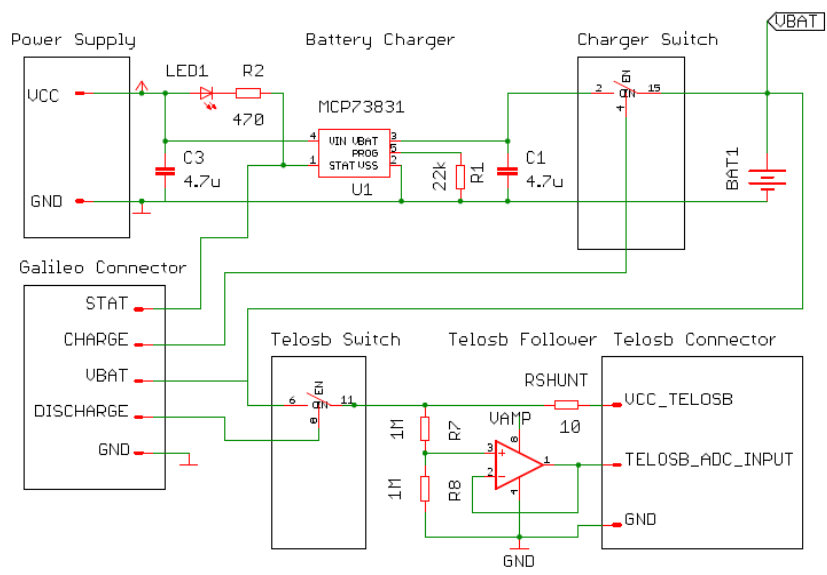

Fig.2. Recharging board with inputs/outputs (connectors) for the Galileo board, Telosb and the power supply. 


\section{B. Galileo Board}

Galileo Board [37] is powerful platform featuring a 32-bit microprocessor that presents an extraordinary capacity of data storage, basically because it is equipped with a large RAM memory and a SD flash memory. The Galileo is connected to the Telosb basestation through the USB port and it can receive and save data packets using this link.

\section{Temperature chamber}

The ambient temperature affects significantly the battery operation. In order to ensure that the temperature was constant during all the charge/discharge cycles, the batteries were put inside a temperature chamber. This chamber has an accuracy of $+/-0.8^{\circ} \mathrm{C}$ in the range $[-40,-25]^{\circ} \mathrm{C}$ and of $+/-0.5^{\circ} \mathrm{C}$ in the interval $[-25,70]{ }^{\circ} \mathrm{C}$. Three different temperatures were selected: $-16^{\circ} \mathrm{C}, 20^{\circ} \mathrm{C}$ and $45^{\circ} \mathrm{C}$. At each temperature, a group of different charge/discharge cycles was collected.

\section{SOH MODELS}

\section{A. Data collection}

Battery parameters that can be sampled directly are: V_high, V_low, Vcc_high, Vcc_low, ambient temperature and number of charge/discharge cycles. Where, V_high and V_low are the sampled battery voltages when the mote is at high power and low power consumption states, respectively. Vcc_high and Vcc_low correspond to the microcontroller internal supply voltage at the two different power levels. Therefore, the saved data files are composed of six columns, containing the values of these parameters. Each row in these files forms a vector that comprises the values of the six parameters taken at the same time.

After the acquisition of these experimental data samples, some other derived parameters can be extracted. For example, I_low and I_high, the mote's currents drained at low and high power states, can be calculated using the voltages and the shunt resistor. With the currents and voltages, it is also possible to estimate the battery internal resistance as it is expressed in Eq. 2. Other significant parameters that can provide relevant information are the SoC [7] and the initial battery voltage at each trace $V_{-}$ini [5]. The complete list of parameters considered is:

- V_high, V_low, I_high, I_low: Voltages and currents at high and low power states.

- V_ini: Initial battery voltage at each trace.

- T: Ambient temperature.

- N_c: Number of charge/discharge cycles.

- R: Battery internal resistance.

- SoC: State of Charge.

- Slope_V, Slope_I and Slope_R: Slopes of the voltage, current and resistance traces.

- V_dif_slope and V_dif_slope_mean: The first parameter is the difference in the slope calculated with only 14 samples or with 70 samples. The second one is the mean of this parameter throughout the same trace.

- V_max, V_var, V_range: Voltage maximum, variance and range for a complete trace.

During the data gathering process, three batteries of the same type were monitored at three different ambient temperatures $\left(45^{\circ} \mathrm{C}, 20^{\circ} \mathrm{C}\right.$ and $\left.-16^{\circ} \mathrm{C}\right)$. Three test-benches were running in parallel in an autonomous way to collect all the programmed data traces. Once all the experimental files were collected, the rest of indirect parameters were calculated off-line, processing the data samples included in the stored files.

When the data collection begins, it is important to save properly the initial data trace, which contains the samples of the first complete discharge cycle of the battery at $20^{\circ} \mathrm{C}$. This trace represents the maximum autonomy of the battery and it is considered as the $100 \%$ of its autonomy. Comparing the length of the initial trace with the length of subsequent data traces, the real SoH of the battery at a given time can be calculated. Thus, the real $\mathrm{SoH}$ at a certain instant is computed off-line comparing the durations of these discharge cycles.

Although the data traces duration is affected by temperature, number of charge/discharge cycles, depth of discharge and other battery parameters, the saved data traces comprise several hundred samples per trace. Hence, a random selection of samples at each trace was made with the aim of reducing the total amount of data and the complexity of its processing. In this way, only 250 data samples were selected from each data trace. It should be noticed that each sample is a vector that comprises the values of all the battery parameters sampled at the same instant. One fifth of the samples come from the final part of the trace, where the battery voltage drops quickly, because an overrepresentation of this non-linear part provides useful information to obtain more accurate models.

\section{B. Preprocessing}

Initially, a bilinear Butterworth filter is applied to smooth the collected data traces and to reduce the effect of the dispersion of measurements and noise. Some of the saved parameters may not provide relevant information and they can be discarded. In this context, the selection of those parameters that provide more relevant information is made applying the correlation-based algorithm [38] and the greedy hillclimbing method based on a local search [39]. The results obtained are:

- V_high, I_high, V_max and V_range are discarded because they are strongly correlated with other parameters and the information they provide is redundant.

- Parameters that are not highly correlated with the real SoH are also excluded. This group includes: SoC, Slope_V, Slope_I, Slope_R, V_dif_slope and V_dif_slope_mean.

- As a result, the final list of selected parameters is: V_low, I_low, R, T, N_c, V_ini and V_var.

\section{Model generation}

Models have been developed using the data mining software Weka [40]. Two families of models have been considered: regressions and neural networks. In this process, only data coming from two batteries were used and $10 \%$ of the data samples were kept for cross-validating the models generated. This means that in Weka 10 folds were selected to verify the models. The traces from the third battery were reserved to validate the models.

\section{1) Regressions}

In this category two different algorithms for generating the models have been used: least squares and linear regression. A first approach based on the well-known least squares algorithm makes use of the Weka least squared regression 
method. This procedure provides different regression functions using random subsamples of the data. After that, the method selects the least squared regression function with lowest median squared error. Applying the Weka software with this method to input data traces, the resulting function is:

$$
\begin{aligned}
\mathrm{SoH}=-0.0065 & \cdot V_{\text {low }}+0.3608 \cdot I_{\text {low }}-0.0001 \cdot T \\
& -0.0028 \cdot R-0.0004 \cdot N_{c} \\
& +0.0307 \cdot V_{\text {ini }}-16.3266 \cdot V_{\text {variance }} \\
& +1.4583
\end{aligned}
$$

A second linear model with the same parameters can be generated considering the common linear regression. The implementation of this method in the Weka software uses the Akaike information criterion to estimate the relative quality of the models for a certain data set. The obtained function applying Weka with this method is:

$$
\begin{aligned}
\text { SoH }=-0.0027 & \cdot V_{\text {low }}+2.1815 \cdot I_{\text {low }}-0.0007 \cdot T \\
& -0.0163 \cdot R-0.0009 \cdot N_{c}+0.2882 \\
& \cdot V_{\text {ini }}-11.0923 \cdot V_{\text {variance }} \\
& +0.1596
\end{aligned}
$$

\section{2) Neural Networks}

As a second approach, models based on the multilayer perceptron (MLP) [41] have been also considered. These models have already been successfully applied to the development of battery models for electric vehicles [27][28]. Although, neural networks are more complex than linear regressions, it should be noticed that the main complexity appears during the model generation in the training step. Thus, these models can be trained on a personal computer using the experimental data traces, and then, once the MLP coefficients are obtained, it can be programmed and executed on WSN motes. During the training step in the Weka software 500 epochs and the sigmoid activation function were used. The expression of the five-neurons network obtained is:

$$
\begin{aligned}
& n_{1}=\frac{1}{1+e^{-\left(k 11 \cdot V_{\text {low }}+k 12 \cdot I_{\text {low }}+k 13 \cdot T+k 14 \cdot R+k 15 \cdot \mathrm{N}_{\mathrm{c}}+k 16 \cdot V_{\text {ini }}+k 17 \cdot V_{\text {var }}+t h 1\right)}} \\
& n_{2}=\frac{1}{1+e^{-\left(k 21 \cdot V_{\text {low }}+k 22 \cdot I_{\text {low }}+k 23 \cdot T+k 24 \cdot R+k 25 \cdot \mathrm{N}_{\mathrm{c}}+k 26 \cdot V_{\text {ini }}+k 27 \cdot V_{\text {var }}+t h 2\right)}} \\
& n_{3}=\frac{1}{1+e^{-\left(k 31 \cdot V_{\text {low }}+k 32 \cdot I_{\text {low }}+k 33 \cdot T+k 34 \cdot R+k 35 \cdot \mathrm{N}_{\mathrm{c}}+k 36 \cdot V_{\text {ini }}+k 37 \cdot V_{\text {var }}+t h 3\right)}} \\
& n_{4}=\frac{1}{1+e^{-\left(k 41 \cdot V_{\text {low }}+k 42 \cdot I_{\text {low }}+k 43 \cdot T+k 44 \cdot R+k 45 \cdot \mathrm{N}_{\mathrm{c}}+k 46 \cdot V_{\text {ini }}+k 47 \cdot V_{\text {var }}+t h 4\right)}} \\
& \text { SoH }=t h 5+k 51 \cdot n 1+k 52 \cdot n 2+k 53 \cdot n 3+k 54 \cdot n 4
\end{aligned}
$$

where the coefficients are:

$\mathrm{k}_{11}=0.19118 ; \mathrm{k}_{12}=-0.54735 ; \quad \mathrm{k}_{13}=0.55937$;

$\mathrm{k}_{14}=-0.04410 ; \mathrm{k}_{15}=0.47650 ; \mathrm{k}_{16}=-0.56882$;

$\mathrm{k}_{17}=2.32554 ; \mathrm{th}_{1}=0.53997$;

$\mathrm{k}_{21}=0.09869 ; \mathrm{k}_{22}=-0.23333 ; \mathrm{k}_{23}=-0.32485$;

$\mathrm{k}_{24}=0.03691 ; \mathrm{k}_{25}=-0.29316 ; \mathrm{k}_{26}=0.00815$;

$\mathrm{k}_{27}=-3.72451 ; \quad \mathrm{th}_{2}=-5.09052 ;$

$\mathrm{k}_{31}=0.17222 ; \mathrm{k}_{32}=-0.36228 ; \quad \mathrm{k}_{33}=-1.49284$;

$\mathrm{k}_{34}=0.09042 ; \mathrm{k}_{35}=-0.60896 ; \quad \mathrm{k}_{36}=-0.18619$;

$\mathrm{k}_{37}=1.03222 ; \mathrm{th}_{3}=0.17275$;

$\mathrm{k}_{41}=-0.48555 ; \mathrm{k}_{42}=1.26417 ; \mathrm{k}_{43}=1.66614 ;$

$\mathrm{k}_{44}=-0.00248 ; \quad \mathrm{k}_{45}=-0.33934 ; \quad \mathrm{k}_{36}=-0.83581$;

$\mathrm{k}_{37}=2.48200 ; \mathrm{th}_{4}=-0.76316$; $\mathrm{k}_{51}=-0.97639 ; \quad \mathrm{k}_{52}=2.00500 ; \quad \mathrm{k}_{53}=-0.75240 ;$

$\mathrm{k}_{54}=-0.50034 ; \quad \mathrm{th}_{5}=0.89178$;

\section{Results AND Discussions}

\section{A. Model validation}

Initially, the models have been evaluated using a subset of the data traces, which have not been used during the models generation. This subset is taken from the initial data traces and comprises a $10 \%$ of these traces. Table I summarizes the cross-validation of the models using these reserved samples.

TABLE I

MEAN RESULTS OF THE PROPOSED MODELS ON THE ORIGINAL MEASURES

\begin{tabular}{lccccc}
\hline \hline Model & Correlation & $\begin{array}{l}\text { Absolute } \\
\text { error } \\
\text { (SoH) }\end{array}$ & $\begin{array}{l}\text { Squared } \\
\text { error } \\
\text { (SoH) }\end{array}$ & $\begin{array}{l}\text { Relative } \\
\text { abs } \\
\text { error(\%) }\end{array}$ & $\begin{array}{l}\text { Relative } \\
\text { squared } \\
\text { error(\%) }\end{array}$ \\
\hline $\begin{array}{l}\text { Least } \\
\text { squares }\end{array}$ & 0.98 & 0.01 & 0.03 & 11.21 & 19.47 \\
\hline $\begin{array}{l}\text { Linear } \\
\text { regression }\end{array}$ & 0.99 & 0.01 & 0.01 & 7.99 & 9.51 \\
\hline $\begin{array}{l}\text { Multilayer } \\
\text { perceptron }\end{array}$ & 1 & 0.001 & 0.001 & 0.70 & 0.82 \\
\hline \hline
\end{tabular}

In Fig. 3 and Fig. 4 it is shown the evolution of the real SoH against the number of charge/discharge cycles for the two batteries at different temperatures. These graphs compare the SoH estimated using the three models considered. The results obtained, shown in Table I, demonstrate the suitability of the proposed models and reveal that the model that best fits on average the real SoH is the MLP. On the other hand, results in Table II breaks down the performance of each model considering in every case only the cycles taken at one particular temperature. Comparing these results, the first conclusion that can be drawn is that the MLP behaves significantly better at $-16^{\circ}$, mainly due to the nonlinear fall of the $\mathrm{SoH}$ at this temperature. At the rest of temperatures MLP continues performing better but the difference is not as pronounced, since the shape of these graphs is more linear.

TABLE II.

MEAN RESULTS OF THE PROPOSED MODELS ON THE ORIGINAL MEASURES AT DIFFERENT TEMPERATURES.

\begin{tabular}{ccccccc}
\hline \hline Model & Temp. & $\begin{array}{l}\text { Corre- } \\
\text { lation }\end{array}$ & $\begin{array}{l}\text { Absolute } \\
\text { error } \\
\text { (SoH) }\end{array}$ & $\begin{array}{l}\text { Squared } \\
\text { error } \\
\text { (SoH) }\end{array}$ & $\begin{array}{l}\text { Relative } \\
\text { abs } \\
\text { error } \\
\text { (\%) }\end{array}$ & $\begin{array}{l}\text { Relative } \\
\text { squared } \\
\text { error } \\
\text { (\%) }\end{array}$ \\
\hline $\begin{array}{c}\text { Least } \\
\text { squares }\end{array}$ & $45^{\circ}$ & 0.99 & 0.003 & 0.01 & 2.57 & 8.54 \\
\hline $\begin{array}{c}\text { Linear } \\
\text { regression }\end{array}$ & $45^{\circ}$ & 0.99 & 0.003 & 0.005 & 2.75 & 3.60 \\
\hline $\begin{array}{c}\text { Multilayer } \\
\text { perceptron }\end{array}$ & $45^{\circ}$ & 0.99 & 0.001 & 0.001 & 0.89 & 1.10 \\
\hline $\begin{array}{c}\text { Least } \\
\text { squares }\end{array}$ & $20^{\circ}$ & 0.99 & 0.001 & 0.002 & 4.49 & 5.91 \\
\hline $\begin{array}{c}\text { Linear } \\
\text { regression }\end{array}$ & $20^{\circ}$ & 0.99 & 0.002 & 0.004 & 5.56 & 6.06 \\
\hline $\begin{array}{c}\text { Multilayer } \\
\text { perceptron }\end{array}$ & $20^{\circ}$ & 0.99 & 0.001 & 0.001 & 2.60 & 3.00 \\
\hline $\begin{array}{c}\text { Least } \\
\text { squares }\end{array}$ & $-16^{\circ}$ & 0.98 & 0.01 & 0.017 & 12.74 & 18.99 \\
\hline $\begin{array}{c}\text { Linear } \\
\text { regression }\end{array}$ & $-16^{\circ}$ & 0.99 & 0.008 & 0.01 & 10.17 & 11.27 \\
\hline $\begin{array}{c}\text { Multilayer } \\
\text { perceptron }\end{array}$ & $-16^{\circ}$ & 1 & 0.0007 & 0.001 & 0.84 & 0.92 \\
\hline \hline
\end{tabular}



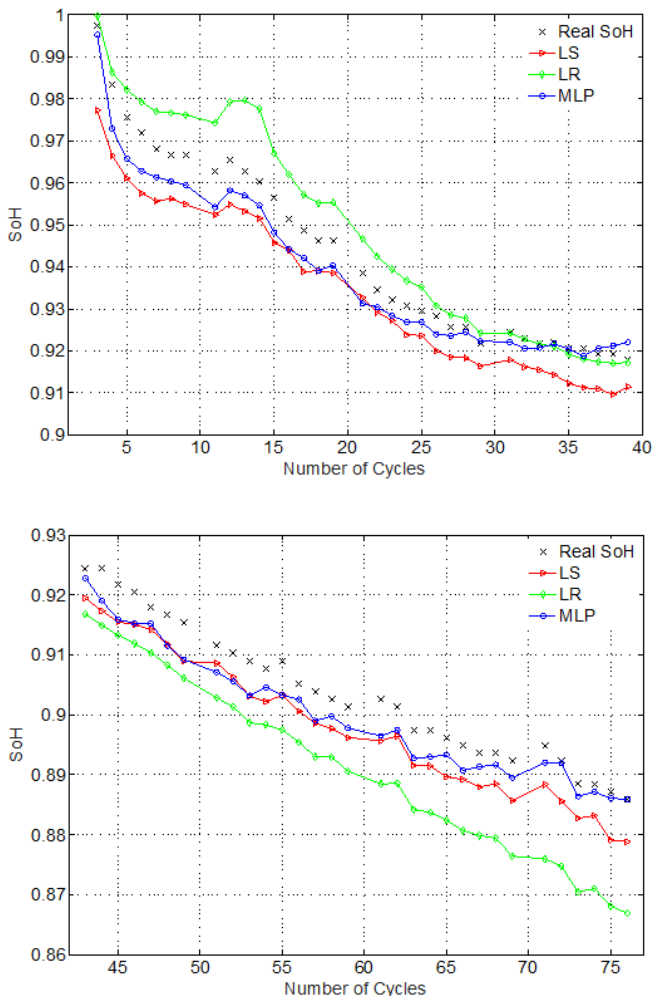

b)

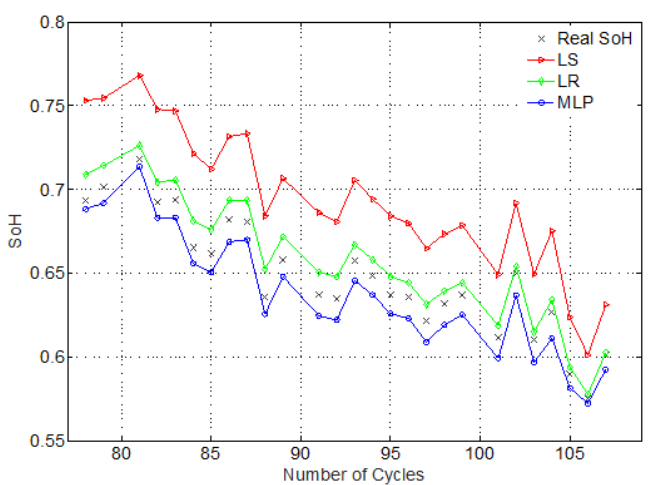

Fig. 3. First battery SoH estimation using the three models generated: LS (least squares), LR (linear regression) and MLP (multilayer perceptron). The data traces were taken at a constant temperature of: a) $45^{\circ} \mathrm{C}$, b) $20^{\circ} \mathrm{C}$ and c) $-16^{\circ} \mathrm{C}$.

It should be noticed that in Fig. 4 a) an unusual effect in the performance of the second battery occurs. As it can be seen, in this graph the battery $\mathrm{SoH}$ increases with the number of cycles, being its evolution opposite to the expected behavior. This effect is due to the sequence of applied temperatures. For the second battery, the operating temperatures during each range of charge/discharge cycles were:

$1^{\circ}$. In the interval from 1 to 40 cycles, shown in Fig. 4 b), cycles were taken at $20^{\circ} \mathrm{C}$.

$2^{\circ}$. In the interval from 40 to 80 cycles, shown in Fig. 4 c), cycles were taken at $-16^{\circ} \mathrm{C}$.

$3^{\circ}$. In the interval from 80 to 100 cycles, shown in Fig. 4 a), cycles were taken at $45^{\circ} \mathrm{C}$.

It is known that extremely low temperatures strongly affect the battery performance [42]. In fact, this is something that is reflected in Fig. 4 c), where the battery performance declines enormously in a short interval of cycles. The rapid degradation suffered is in the order of the $40 \%$ of the initial SoH, which is in accordance with some previous references [43]. Then, when the temperature changes to $45^{\circ} \mathrm{C}$ in Fig. 4 a), again there is an abrupt variation of the battery performance that increases in more than a $10 \%$. However, the battery does not fully recover the initial performance and there is tendency to increase the SoH slowly that accounts for the slight SoH improvement in this graph. In contrast, this effect does not appear in the first battery due to the different sequence of temperatures applied:

$1^{\circ}$. In the interval from 1 to 40 cycles, shown in Fig. 3 a), cycles were taken at $45^{\circ} \mathrm{C}$.

$2^{\circ}$. In the interval from 40 to 76 cycles, shown in Fig. $3 \mathrm{~b}$ ), cycles were taken at $20^{\circ} \mathrm{C}$.

$3^{\circ}$. In the interval from 77 to 110 cycles, shown in Fig. 3 c), cycles were taken at $-16^{\circ} \mathrm{C}$.

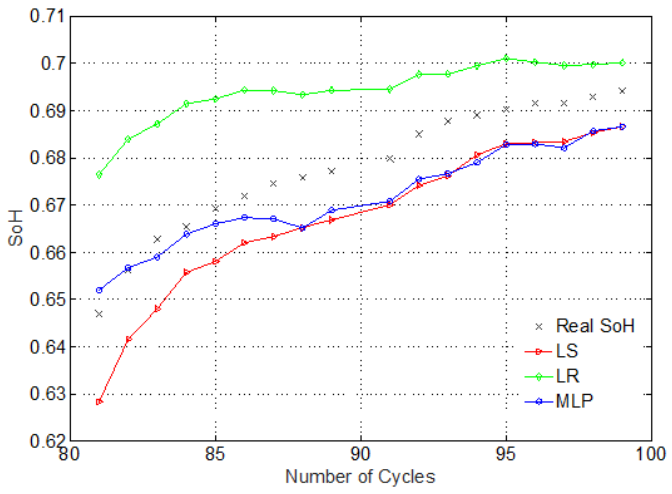

a)

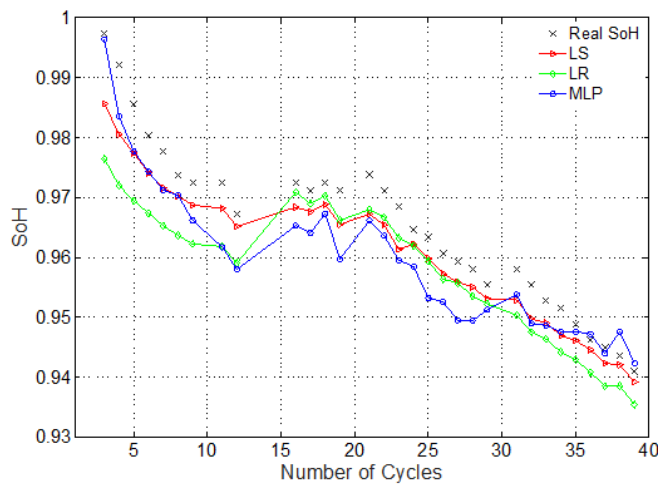

b)

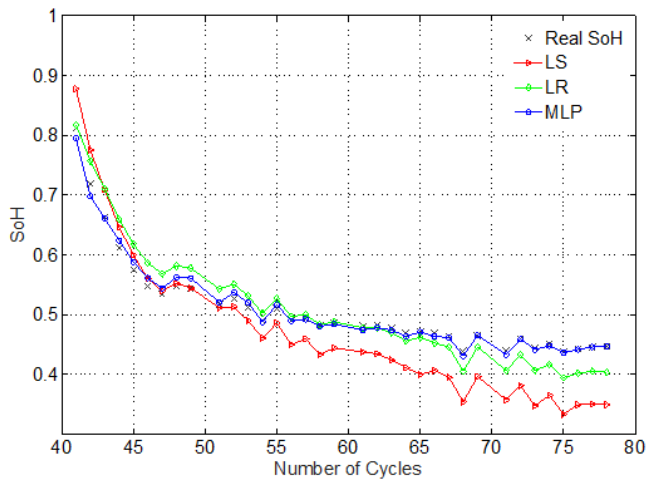

c)

Fig. 4. Second battery $\mathrm{SoH}$ estimation using the three models generated: LS (least squares), LR (linear regression) and MLP (multilayer perceptron). The data traces were taken at a constant temperature of: a) $45^{\circ} \mathrm{C}$, b) $20^{\circ} \mathrm{C}$ and c) $-16^{\circ} \mathrm{C}$. 
Apart from the results obtained with the data crossvalidation using traces from the two first batteries, another experiment has been conducted to determine the correctness of the models. In this second test, the models were applied to data traces coming from a third battery of the same type. Results showing the SoH estimation for the third battery are presented in Fig. 5.

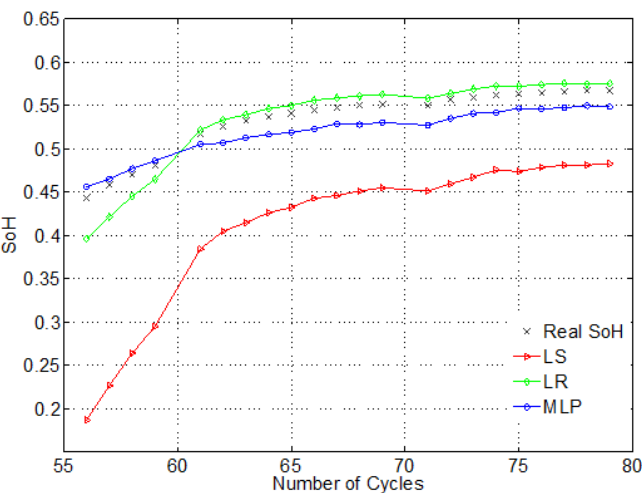

a)

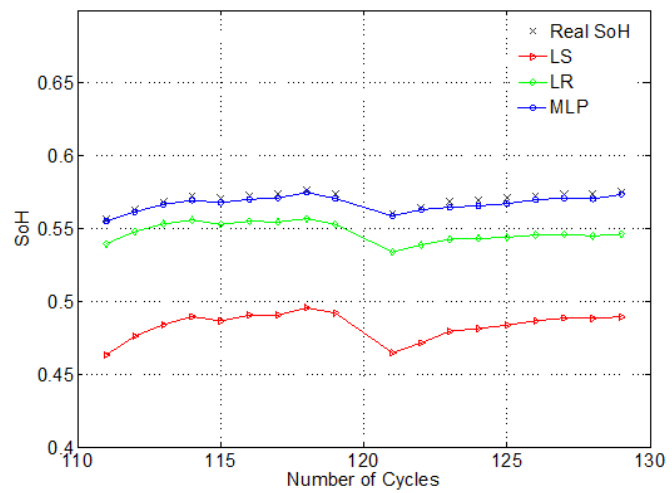

b)

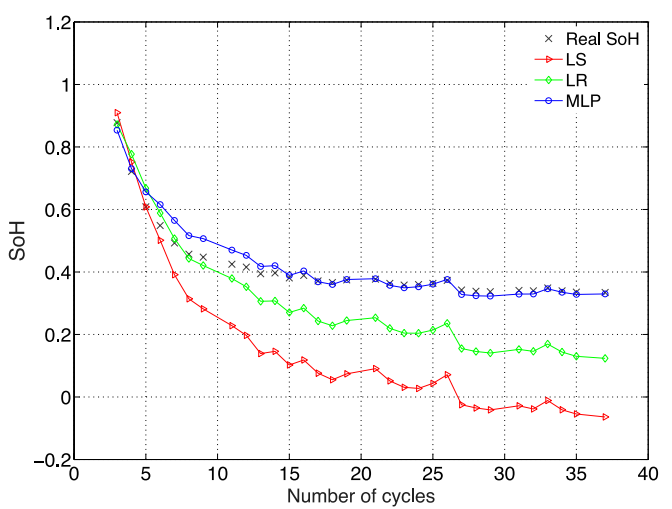

c)

Fig. 5. Third battery SoH estimation of the three models generated using LS (least squares), LR (linear regression) and MLP (multilayer perceptron). The data traces were taken at a constant temperature of: a) $45^{\circ} \mathrm{C}$, b) $20^{\circ} \mathrm{C}$ and c) $-16^{\circ} \mathrm{C}$.

As it can be seen, in general the models accurately fit the real $\mathrm{SoH}$ and they can predict its evolution. It can be also concluded that the model that best fits the SoH line regardless of the temperature is again the MLP. In this case, the same effect observed in the second battery occurs due to the selected sequence of temperatures:

$1^{\circ}$. In the interval from 1 to 40 cycles, shown in Fig. 5 c), cycles were taken at $-16^{\circ} \mathrm{C}$. $2^{\circ}$. In the interval from 55 to 80 cycles, shown in Fig. 5 a), cycles were taken at $45^{\circ} \mathrm{C}$.

$3^{\circ}$. In the interval from 110 to 130 cycles, shown in Fig. 5 b), cycles were taken at $20^{\circ} \mathrm{C}$.

Thus, in Fig. 5 c) there is a rapid initial degradation of the battery performance and a slow improvement during the cycles in Fig. 5 a) and b).

Finally, the last experiment that has been conducted involves a fourth battery, tested at $25^{\circ} \mathrm{C}$, and some variations in the program executed in the mote. In this case, we continue applying the same mathematical models but the program that the mote runs has been changed varying the duty cycle. The program is composed of the same states that the one presented in section III, but the six seconds delay in state 4 is removed. Thus, the mote is approximately $300 \mathrm{~ms}$ awake in a high power mode and 700 ms asleep in a power down state. Results of the predicted SoH for this battery are depicted in Fig. 6 and the numerical results are provided in Table III. As it can be seen, the precision of the estimated $\mathrm{SoH}$ in these cases remains practically the same and we can conclude that the program duty cycle is not a critical factor for the SoH estimation.

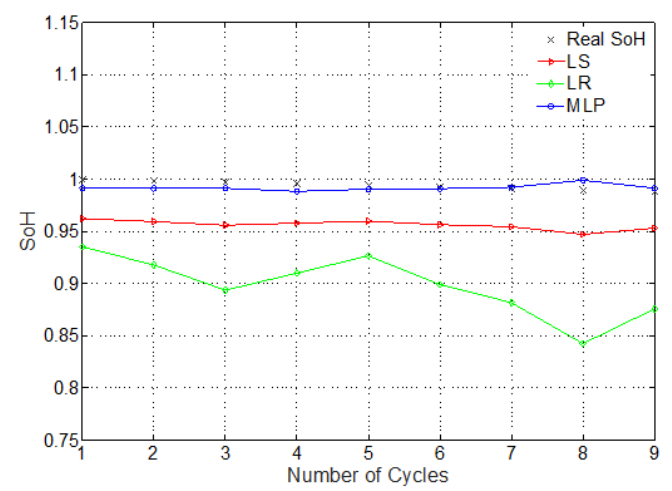

Fig. 6. Fourth battery $\mathrm{SoH}$ estimation at $25^{\circ} \mathrm{C}$ using the three proposed models.

TABLE III.

MEAN RESULTS OF THE PROPOSED MODELS FOR A DIFFERENT PROGRAM.

\begin{tabular}{cccccc}
\hline \hline Model & Correlation & $\begin{array}{l}\text { Absolute } \\
\text { error } \\
\text { (SoH) }\end{array}$ & $\begin{array}{l}\text { Squared } \\
\text { error } \\
\text { (SoH) }\end{array}$ & $\begin{array}{l}\text { Relative } \\
\text { abs error } \\
\text { (\%) }\end{array}$ & $\begin{array}{l}\text { Relative } \\
\text { squared } \\
\text { error (\%) }\end{array}$ \\
\hline $\begin{array}{c}\text { Least } \\
\text { squares }\end{array}$ & 1 & 0.037 & 0.03 & 11.09 & 9.61 \\
\hline $\begin{array}{c}\text { Linear } \\
\text { regression }\end{array}$ & 1 & 0.095 & 0.09 & 8.29 & 10.51 \\
\hline $\begin{array}{l}\text { Multilayer } \\
\text { perceptron }\end{array}$ & 1 & 0.005 & 0.006 & 1.58 & 1.54 \\
\hline \hline
\end{tabular}

\section{B. Comparison with other methods}

In this subsection we compare the previous models with an additional model obtained using the method proposed in reference [14]. The application of this method starts with the acquisition of battery parameters during the charging stage at constant voltage. With these data traces, it can be obtained an experimental estimation of the equivalent time constant of the battery, which is highly correlated with the $\mathrm{SoH}$, as it is demonstrated in [14]. In our case, we have determined these values from the experimental battery data traces, using the charging time of every charge/discharge cycle and approximating the equivalent time constant to the $63 \%$ of this 
time. We have implemented the model applying the least squares regression, as it is proposed in [14], to the data cycles of the second battery taken at $20^{\circ} \mathrm{C}$. With this model, we have estimated the error of predicting the $\mathrm{SoH}$ using the data coming from the first battery at $20^{\circ} \mathrm{C}$ and $40^{\circ} \mathrm{C}$. Fig. 7 shows that the cycles of the first battery taken at $20^{\circ} \mathrm{C}$ fit the linear model and are mostly concentrated within an error bound of $\pm 1.5 \%$, as it was expected from results presented in [14]. However, the cycles taken at $45^{\circ} \mathrm{C}$ do not match the same linear model because the temperature affects the charging time and this parameter is not considered in the model generation, according the authors, it is a future work. In reference [14], the generalization of the model to other temperatures is contemplated as future work. Table IV summarizes the obtained results using the data from the first battery at three temperatures: $20^{\circ} \mathrm{C}, 45^{\circ} \mathrm{C}$ and $-16^{\circ} \mathrm{C}$. As it can be observed, this method provides only precise predictions when the temperature does not change $\left(20^{\circ} \mathrm{C}\right)$. However, results get worse at other temperatures. Thus, this comparison has demonstrated the suitability of the method presented in [14] to provide accurate models for predicting the $\mathrm{SoH}$ but only at constant temperature. In any case, the results obtained at $20^{\circ} \mathrm{C}$ are comparable to the results of the linear models that we have proposed, however the MLP model outperforms these results regardless the temperature considered.

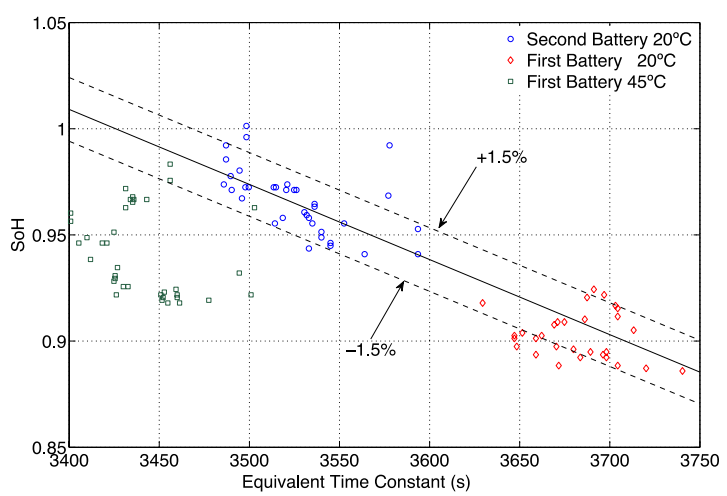

Fig. 7. Linear model that correlates $\mathrm{SoH}$ and the equivalent time constant obtained using the method in [14] with data coming from the first and the second batteries.

TABLE IV.

MEAN RESULTS OF THE MODEL IN [14] GENERATED AT 20ㄷ FOR DATA COMING FROM THE FIRST BATTERY AT TWO DIFFERENT TEMPERATURES.

\begin{tabular}{cccccc}
\hline \hline Temperature & Correlation & $\begin{array}{l}\text { Absolute } \\
\text { error } \\
\text { (SoH) }\end{array}$ & $\begin{array}{l}\text { Squared } \\
\text { error } \\
\text { (SoH) }\end{array}$ & $\begin{array}{l}\text { Relative } \\
\text { abs } \\
\text { error } \\
\text { (\%) }\end{array}$ & $\begin{array}{l}\text { Relative } \\
\text { squared } \\
\text { error } \\
(\%)\end{array}$ \\
\hline $20^{\circ} \mathrm{C}$ & 1 & 0.013 & 0.015 & 3.00 & 2.88 \\
\hline $45^{\circ} \mathrm{C}$ & 1 & 0.09 & 0.092 & 9.91 & 9.20 \\
\hline$-16^{\circ} \mathrm{C}$ & 0.45 & 0.781 & 0.791 & 82.6 & 61.7 \\
\hline
\end{tabular}

\section{Models implementation}

The proposed models have been implemented on Telosb motes using TinyOS and the $\mathrm{C}$ language floating-point library math.h to evaluate the computational cost. In the case of the MLP model, the input data normalization is added to the global cost. On the other hand, the data acquisition process, the filters application or de-normalization of the MLP output is not considered because the assessment is only focused on the computational cost of the models. Measurements in terms of internal clock cycles were performed with the TinyOS Counter32khz32C component, which implements a $32 \mathrm{kHz}$ counter that relies on an internal microcontroller's timer.

The obtained result for both linear models is the same and it is equal to 138 cycles, measured with the $32 \mathrm{kHz}$ timer. In the case of MLP the number of cycles is 885 cycles, which represents a significant increase with respect to the linear case, due to the higher complexity of its mathematical expression. The energy consumption can be calculated considering the mote operating conditions and the number of cycles. It is assumed that the Telosb mote has a current consumption of $2.3 \mathrm{~mA}$ (MSP430@4 MHz) during the execution of the models. Taking into account the current consumption, the supply voltage and the time required during the models execution, the energy consumed can be calculated. Table V shows the cost of calculating the SoH in Telosb motes in terms of time and energy. We can compare the energy consumed in the calculation of the models with other typical actions that a mote carries out during its operation. For example, a packet transmission using the maximum power level with 18 Bytes in the payload consumes $2.16 \mathrm{~mJ}$, the reception of a packet is $2.13 \mathrm{~mJ}$ and writing 1 Byte in the flash memory represents $0.0349 \mathrm{~mJ}$ [44]. As a result, we can conclude that the energy consumption of calculating the SoH with the proposed models is much lower than transmitting or receiving a wireless packet.

TABLE V.

COMPUTATIONAL AND ENERGY COST OF THE MODELS.

\begin{tabular}{lll}
\hline \hline Model & Time $(\mathbf{m s})$ & Energy $(\mathbf{m J})$ \\
\hline linear & 4.211 & 0.031 \\
\hline MLP & 27 & 0.204 \\
\hline \hline
\end{tabular}

\section{CONCLUSIONS}

In this work we have proposed a methodology for the generation of simple models that can estimate the $\mathrm{SoH}$ of rechargeable batteries in WSN applications. As a first step, the method requires the collection of data traces of the battery parameters under different operating conditions and during the execution of a typical WSN application with different power consumption states. During the generation of the SoH models a specific group of relevant battery parameters was determined. With this group of parameters and the initial data traces the models can be obtained. These models have been validated with a new fresh data coming from other batteries. The conducted tests have proved the validity of the models and the adequacy of the method to provide accurate $\mathrm{SoH}$ models. As it is demonstrated in the results section, the MLP model outperforms the rest of models and accurately fits the $\mathrm{SoH}$ evolution but at the expense of increasing the program computational cost. This cost has been evaluated in terms of time and energy consumption during its execution in a Telosb mote. Results show the suitability of the models for being executed in simple motes and their relatively low consumption compared to other typical actions that the mote commonly executes. As a result, we consider that the proposed models can accurately predict the $\mathrm{SoH}$ with a reasonable increase of 
energy and computational cost. This work can be extended in the future considering different batteries and the inclusion of energy harvesting systems.

\section{REFERENCES}

[1] J. Alberola, J. Pelegri, R. Lajara, and J. J. Perez, "Solar inexhaustible power source for wireless sensor node," in Proc. IEEE Instrum. Meas.Technol. Conf. (IMTC), May 2008, pp. 657-662.

[2] R. J. Lajara, J. J. Perez-Solano and J. Pelegrí-Sebastia, "A Method for Modeling the Battery State of Charge in Wireless Sensor Networks," in IEEE Sensors Journal, vol. 15, no. 2, pp. 1186-1197, Feb. 2015.

[3] B. Buchli, D. Aschwanden, and J. Beutel, "Battery state-of-charge approximation for energy harvesting embedded systems”, in Wireless Sensor Networks (Lecture Notes in Computer Science), vol. 7772. Berlin, Germany: Springer-Verlag, 2013, pp. 179-196.

[4] V. Quintero, C. Estevez and M. Orchard, "State-of-charge estimation to improve energy conservation and extend battery life of wireless sensor network nodes," 2017 Ninth International Conference on Ubiquitous and Future Networks (ICUFN), Milan, 2017, pp. 153-158.

[5] M. Berecibar, I. Gandiaga, I. Villarreal, N. Omar, J. Van Mierlo, P. Van den Bossche, Critical review of state of health estimation methods of Liion batteries for real applications, Renewable and Sustainable Energy Reviews, Volume 56, April 2016, Pages 572-587, ISSN 1364-0321.

[6] L. Zhao et al., "Energy-aware system design for wireless sensor network,” ACTA Autom. Sinica, vol. 32, no. 6,p. 892, 2006.

[7] R. Lajara, J. Pelegri-Sebastia and J. Perez-Solano, "Method for measuring internal resistance of batteries in WSN," IEEE Sensors 2014 Proceedings, Valencia, 2014, pp. 1984-1987.

[8] Remmlinger, J., Buchholz, M., Meiler, M., Bernreuter, P., \& Dietmayer, K. (2011). State-of-health monitoring of lithium-ion batteries in electric vehicles by on-board internal resistance estimation. Journal of Power Sources, 196(12), 5357-5363.

[9] Cheng Lin, Aihua Tang, Wenwei Wang, A Review of SOH Estimation Methods in Lithium-ion Batteries for Electric Vehicle Applications, Energy Procedia, Volume 75, 2015, Pages 1920-1925, ISSN 1876-6102.

[10] Stancovski, Victor, and Simona Badilescu. "In situ Raman spectroscopic-electrochemical studies of lithium-ion battery materials: a historical overview." Journal of Applied Electrochemistry 44.1 (2014): 23-43.

[11] D. I. Stroe et al., "Diagnosis of lithium-ion batteries state-of-health based on electrochemical impedance spectroscopy technique," 2014 IEEE Energy Conversion Congress and Exposition (ECCE), Pittsburgh, PA, 2014, pp. 4576-4582.

[12] Chiang, Yi-Hsien, Wu-Yang Sean, and Jia-Cheng Ke. "Online estimation of internal resistance and open-circuit voltage of lithium-ion batteries in electric vehicles." Journal of Power Sources 196.8 (2011): 3921-3932.

[13] Ng, Kong Soon, et al. "Enhanced coulomb counting method for estimating state-of-charge and state-of-health of lithium-ion batteries." Applied energy86.9 (2009): 1506-1511.

[14] J. Yang, B. Xia, W. Huang and C. Mi, "On-board state-of-health estimation based on charging current analysis for LiFePO4 batteries,"2017 IEEE Energy Conversion Congress and Exposition (ECCE), Cincinnati, OH, 2017, pp. 5229-5233.

[15] D. Haifeng, et al., "A new SOH prediction concept for the power lithium-ion battery used on HEVs," 2009 IEEE Vehicle Power and Propulsion Conference, Dearborn, MI, 2009, pp. 1649-1653.

[16] Zhen Guo, Xinping Qiu, Guangdong Hou, Bor Yann Liaw, Changshui Zhang, State of health estimation for lithium ion batteries based on charging curves, Journal of Power Sources, Volume 249, 1 March 2014, Pages 457-462, ISSN 0378-7753.

[17] Plett, Gregory L. "Extended Kalman filtering for battery management systems of LiPB-based HEV battery packs: Part 2. Modeling and identification." Journal of power sources 134.2 (2004): 262-276.

[18] D. Haifeng, W. Xuezhe and S. Zechang, "A new SOH prediction concept for the power lithium-ion battery used on HEVs," 2009 IEEE Vehicle Power and Propulsion Conference, Dearborn, MI, 2009, pp. 1649-1653.

[19] Saeed Sepasi, Reza Ghorbani, Bor Yann Liaw, A novel on-board stateof-charge estimation method for aged Li-ion batteries based on model adaptive extended Kalman filter, Journal of Power Sources, Volume 245, 1 January 2014, Pages 337-344.
[20] Chen, Zheng, et al. "Online battery state of health estimation based on genetic algorithm for electric and hybrid vehicle applications." Journal of Power Sources 240 (2013): 184-192.

[21] Liu, Xingtao, et al. "A novel temperature-compensated model for power Li-ion batteries with dual-particle-filter state of charge estimation." Applied Energy 123 (2014): 263-272.

[22] Wang, Yujie, Chenbin Zhang, and Zonghai Chen. "A method for stateof-charge estimation of LiFePO4 batteries at dynamic currents and temperatures using particle filter." Journal of power sources 279 (2015): 306-311.

[23] S. J. Moura, N. A. Chaturvedi and M. Krstić, "PDE estimation techniques for advanced battery management systems - Part II: SOH identification," 2012 American Control Conference (ACC), Montreal, QC, 2012, pp. 566-571.

[24] Moura, S. J., Krstic, M., \& Chaturvedi, N. A. (2012, October). Adaptive PDE observer for battery SOC/SOH estimation. In ASME 2012 5th Annual Dynamic Systems and Control Conference joint with the JSME 2012 11th Motion and Vibration Conference (pp. 101-110). American Society of Mechanical Engineers.

[25] Andre, Dave, et al. "Advanced mathematical methods of SOC and SOH estimation for lithium-ion batteries." Journal of Power Sources 224 (2013): 20-27.

[26] Widodo, Achmad, et al. "Intelligent prognostics for battery health monitoring based on sample entropy." Expert Systems with Applications 38.9 (2011): 11763-11769.

[27] Gong, Q., Li, Y., \& Peng, Z. Power management of plug-in hybrid electric vehicles using neural network based trip modeling. In American Control Conference, 2009. ACC'09, 4601-4606. IEEE.

[28] Sheikhan, M., Pardis, R., \& Gharavian, D.. State of charge neural computational models for high energy density batteries in electric vehicles. Neural Computing and Applications, 2013, 1-10.

[29] H. T. Lin, T. J. Liang and S. M. Chen, "Estimation of Battery State of Health Using Probabilistic Neural Network," in IEEE Transactions on Industrial Informatics, vol. 9, no. 2, pp. 679-685, May 2013.

[30] Eddahech, Akram, et al. "Behavior and state-of-health monitoring of Liion batteries using impedance spectroscopy and recurrent neural networks." International Journal of Electrical Power \& Energy Systems 42.1 (2012): 487-494.

[31] Ho-Ta Lin, Tsorng-Juu Liang and S. M. Chen, "The state-of-health diagnosis of Li-Co batteries with fuzzy identification," Power Electronics and Motion Control Conference (IPEMC), 2012 7th International, Harbin, China, 2012, pp. 2678-2682.

[32] Xtra-power. Rechargeable 3.7V Li-ion Polymer Battery Specification, $40 \mathrm{mAh}$. Available online: http://www.xtra-power.com.hk/Products/Lipolymer/Standard\%20Type/Xtra-power\%20051213(40mAh).pdf (accessed on 5/7/2017)

[33] Telosb Datasheet. Available online: http://www.eecs.harvard.edu/ konrad/projects/shimmer/references/tmot e-sky-datasheet.pdf (accessed on 5/7/2017)

[34] Antonopoulos, C.; Prayati, A; Stoyanova, T.; Koulamas, C.; Papadopoulos, G., "Experimental evaluation of a WSN platform power consumption," Parallel \& Distributed Processing, 2009. IPDPS 2009. IEEE International Symposium on , vol., no., pp.1,8, 23-29 May 2009

[35] Sensirion. Datasheet SHT11 temperature and humidity sensor. Available online:https://www.sensirion.com/fileadmin/user_upload/customers/sens irion/Dokumente/2_Humidity_Sensors/Sensirion_Humidity_Sensors_S HT1x_Datasheet_V5.pdf (accessed on 5/7/2017).

[36] Microchip. Datasheet MCP73831/2 battery charger. Available online: http://ww1.microchip.com/downloads/en/DeviceDoc/21984F.pdf (accessed on 1/10/2016).

[37] Galileo board Datasheet. Available online: http://www.intel.com/content/dam/support/us/en/documents/galileo/sb/g alileo_datasheet 329681_003.pdf (accessed on 5/7/2017).

[38] M. A. Hall. Correlation-based Feature Subset Selection for Machine Learning. Phd thesis. University of Waikato. Hamilton, New Zealand, 1999.

[39] Russell, Stuart J.; Norvig, Peter (2003), Artificial Intelligence: A Modern Approach (2nd ed.), Upper Saddle River, New Jersey: Prentice Hall, pp. 111-114, ISBN 0-13-790395-2.

[40] Hall, M., Frank, E., Holmes, G., Pfahringer, B., Reutemann, P., \& Witten, I. H.. The WEKA data mining software: an update. ACM SIGKDD Explorations Newsletter, 2009, 11(1), 10-18. 
[41] Rumelhart, D. E., Hinton, G. E., \& Williams, R. J. Learning internal representations by error propagation (No. ICS-8506). ICS Report. California Univ San Diego La Jolla inst for cognitive science, 1985.

[42] Ji, Yan, Yancheng Zhang, and Chao-Yang Wang. Li-ion cell operation at low temperatures.Journal of The Electrochemical Society 160.4 (2013): A636-A649.

[43] Wang, Chao-Yang, et al. Lithium-ion battery structure that self-heats at low temperatures. Nature 529.7587 (2016): 515.

[44] Amiri, M. Measurements of energy consumption and execution time of different operations on Tmote Sky sensor nodes. Phd, Masarykova Univerzita, Fakulta informatiky. 